\title{
Comportamiento de ánodos de sacrificio en la protección de aluminio NV-5083 en agua de mar, bahía de Valparaíso
}

\author{
Rosa Vera $^{1}$, Carla Bobadilla ${ }^{1} \&$ Alejandro Madrid ${ }^{1}$ \\ ${ }^{1}$ Laboratorio de Corrosión, Instituto de Química, Pontificia Universidad Católica de Valparaíso \\ Avda. Brasil 2950, Casilla 4059, Valparaíso, Chile
}

\begin{abstract}
RESUMEN. Se evalúa comparativamente el comportamiento de aleaciones de aluminio y cinc, como ánodos de sacrificio en la protección catódica de cascos de aluminio NV-5083 en agua de mar de la bahía de Valparaíso. Para ello se confeccionaron probetas de la aleación de aluminio empleado en cascos de lanchas y ánodos de Al-Zn-In, Al-Zn-Sn y Zn. Los comportamientos electroquímicos se evaluaron mediante curvas de polarización y potencial de corrosión en función del tiempo. Paralelamente, se diseñaron pares galvánicos de aleación del casco-ánodo de sacrificio, los cuales se sumergieron en el electrolito durante 16 días, determinándose la pérdida en peso y la evolución del potencial a circuito abierto de cada material. Los resultados muestran que los ánodos de base aluminio pueden proteger catódicamente el casco de aluminio NV-5083 en agua de mar, pero no logran superar el horizonte económico y de vida útil de los ánodos de cinc, debido a que presentan un mayor desgaste para la protección de igual área de aluminio.
\end{abstract}

Palabras clave: corrosión, aluminio, protección catódica, ánodo de sacrificio, par galvánico, agua de mar.

\section{Behavior of sacrifice anodes in the aluminium NV-5083 protection in seawater, Valparaíso bay}

\begin{abstract}
The behavior of aluminum and zinc alloys were evaluated and compared as sacrifice anodes in cathodic protection of aluminum NV-5083 hulls in seawater in Valparaíso bay. Samples of the aluminum alloy used to manufacture boat hulls and Al-Zn-In, Al-Zn-Sn, and Zn anodes were made. Electrochemical behavior was evaluated by means of polarization curves and corrosion potential as function of time. In parallel, galvanized pairs (boat hull-sacriice anode), were designed and immersed in the electrolyte for 16 days, determining the weight loss and evolution of the open circuit potential for each material. The results show that the aluminum based anodes provide the NV-5083 aluminum hull cathodic protection in seawater. However, they do not exceed the economical horizon and useful life of zinc anodes, because the aluminum based anodes present higher corrosion for the protection of a similar exposed area of aluminum.
\end{abstract}

Key words: corrosion, catodic protection, sacrifice anode, galvanic couple, seawater.

Autor corresponsal: Rosa Vera (rvera@ucv.cl)

\section{INTRODUCCIÓN}

La corrosión acuosa es un fenómeno de naturaleza electroquímica y su característica principal es que ocurre en presencia de un electrolito, como por ejemplo, agua de mar. Este proceso generalmente es peligroso, no solo por la pérdida de material, sino también por tratarse en algunos casos de un ataque localizado que da origen a picaduras de distintas profundidades sobre el metal (Breslin et al., 1991; Latona et al., 2001).

Un caso típico de esta forma de corrosión es el que sufren los cascos de las embarcaciones, generándose un circuito electroquímico o pila de corrosión, que trae como consecuencia una pérdida progresiva de espesor en algunas zonas del metal del casco (Cabrera et al., 1993).

Las pilas de corrosión se encuentran en todo el casco y sus efectos son más o menos intensos según sea la diferencia de potencial entre los materiales que lo constituyen. Algunos ejemplos de estas pilas son planchas de acero de diferente composición, uniones 
de planchas con soldadura, temperatura y aireación diferencial entre la zona de flotación y el fondo, daños mecánicos al casco, efectos buque-muelles, etc. (Bradford, 1993).

De ahí la importancia de controlar el avance de la corrosión, siendo uno de los métodos utilizados la protección catódica por ánodos de sacrificio. Esta metodología consiste básicamente en imponer una corriente continua que lleve al casco de la embarcación a un rango de potencial inmune en agua de mar, de tal manera que cualquier reacción de disolución de las aleaciones que lo componen resulte termodinámicamente imposible (Maguire \& Thompson, 1996; Batt, 2002).

El diseño de un sistema por ánodos de sacrificio debe considerar varios aspectos en el material utilizado, entre otros, una adecuada selección de material para la conformación del ánodo, la cantidad y distribución adecuada para lograr la corriente requerida y la definición previa de la vida útil de los ánodos, de forma de alcanzar la protección del metal durante el tiempo deseado (Cordero \& De Paula, 1997; Schramuk \& Klopper, 2005).

El desempeño y conveniencia de los ánodos a utilizar para una aplicación específica, depende de la composición del electrolito, material a proteger, temperatura de operación y densidad de corriente del ánodo (Tighe-Ford \& Dahele, 2000; Eliezer \& Antonyraj, 2001).

En el caso de la protección catódica para cascos de aluminio en agua de mar, se requiere de 10 veces menos corriente que para el acero, debido a las características aislantes de la capa de óxido que se forma sobre la superficie del aluminio. El carácter protector de esta película permite disminuir las densidades de corriente necesarias para proteger el casco, y así, garantizar la protección catódica en áreas susceptibles a corrosión por picado o corrosión por rendijas (Gundersen \& Nisancioglu, 1990).

Los ánodos más utilizados en la protección de cascos de aluminio en agua de mar son de cinc ( $\mathrm{Zn})$ o de aleaciones ternarias del metal, del tipo Al-Zn-X, donde $\mathrm{X}$ es mercurio $(\mathrm{Hg})$, galio $(\mathrm{Ga})$, estaño $(\mathrm{Sn})$ o indio (In) (Uruchurtu, 1991; British Standards Institution, 1996).

Generalmente, los ánodos de base aluminio presentan una eficiencia mayor que la de los ánodos de cinc en la protección del aluminio en agua de mar (Martínez, 1998). Por ejemplo, al emplear una aleación de Al-In como ánodo de sacrificio, el acoplamiento con el casco a proteger incrementará la disolución de la aleación, contribuyendo a una mejor distribución de corriente. Por lo tanto, esta unión ayudará a la activación de la superficie por el aumento de la corriente catódica de protección. Este comportamiento permitirá que el aluminio se active solo cuando los iones $\mathrm{In}^{-}$se reducen en presencia de iones $\mathrm{Cl}^{-}$(Raja \& Venugopal, 1996).

Por otra parte, en ánodos de aleaciones que contienen $\mathrm{Sn}$ y $\mathrm{Zn}$ los efectos de activación del aluminio dependerán de las condiciones de fundición y de los tratamientos térmicos al que ha sido sometido. En estas condiciones la formación de zonas enriquecidas en $\mathrm{Sn}-\mathrm{Zn}$ en sitios específicos controla el potencial de activación, el ataque de iniciación, los sitios de propagación y la eficiencia del ánodo (Bessone \& Salinas, 1991).

Actualmente, se emplean embarcaciones con casco de aluminio en agua de mar protegidas mediante ánodos de sacrificio de base aluminio. Su uso permite disponer de embarcaciones más ligeras, rápidas y a su vez, con mayor vida útil que las embarcaciones que poseen casco de acero y ánodos de sacrificio a base de cinc (Benedetti et al., 1998; Suriya et al., 2005).

Por lo anterior, el presente trabajo tiene como objetivo general estudiar el comportamiento de distintos ánodos de sacrificio como protección de aluminio NV-5083 en agua de mar, proveniente de la bahía de Valparaíso.

\section{MATERIALES Y MÉTODOS}

La composición química de los materiales utilizados en este estudio: aluminio NV-5083 y los metales empleados como ánodos de sacrificio (aleaciones base aluminio y cinc), se indica en la Tabla 1 . El agua de mar de la bahía de Valparaíso utilizada en los ensayos tenía una salinidad promedio de 34,3 psu, contenido de oxígeno disuelto promedio de $5,6 \mathrm{~mL} \cdot \mathrm{L}^{-1}$ y una temperatura promedio de $13,4^{\circ} \mathrm{C}$.

\section{Ensayos electroquímicos}

Los ensayos electroquímicos comprendieron las determinaciones de potencial de corrosión a circuito abierto (ECA) y curvas de polarización (CP). Para la medida de potencial se utilizó una celda electroquímica convencional con un electrodo de referencia de calomel saturado (ecs) y un electrodo de trabajo de $1 \mathrm{~cm}^{2}$ de superficie, previamente lijado con papel de $\mathrm{SiC}$ de granulometría 400 a 600, conectados a un 
Tabla 1. Composición química de los materiales utilizados.

Table 1. Chemical composition of utilized materials.

\begin{tabular}{|c|c|c|c|c|}
\hline \multirow[b]{2}{*}{ Elemento } & \multicolumn{4}{|c|}{ Composición química (\%) } \\
\hline & Al NV-5083 & Al-Zn-In & Al-Zn-Sn & Zn Mil 18001 \\
\hline $\mathrm{Si}$ & 0,130 & 0,082 & 0,081 & \\
\hline $\mathrm{Fe}$ & 0,240 & 0,101 & 0,100 & 0,001 \\
\hline $\mathrm{Cu}$ & 0,005 & & & 0,001 \\
\hline $\mathrm{Mn}$ & 0,580 & & & \\
\hline $\mathrm{Mg}$ & 4,570 & & & \\
\hline $\mathrm{Cr}$ & 0,100 & & & \\
\hline $\mathrm{Ni}$ & 0,003 & & & \\
\hline $\mathrm{Zn}$ & 0,011 & 4,720 & 4,870 & 99,705 \\
\hline $\mathrm{Ti}$ & 0,019 & & & \\
\hline $\mathrm{Al}$ & 94,342 & 95,073 & 94,922 & 0,230 \\
\hline $\mathrm{Cd}$ & & & & 0,060 \\
\hline $\mathrm{Pb}$ & & & & 0,003 \\
\hline In & & 0,022 & & \\
\hline $\mathrm{Sn}$ & & & 0,021 & \\
\hline Otros & & 0,012 & 0,010 & \\
\hline
\end{tabular}

potenciostato LADEM LA-109. En la realización de las curvas se emplea un alambre de platino de gran área como contraelectrodo.

\section{Ensayos de protección catódica}

En los ensayos de protección catódica se empleó aluminio NV-5083 como cátodo y las respectivas aleaciones y cinc como ánodos de sacrificio. La relación de área de trabajo catódo:ánodo fue de 1:50 y en estas condiciones el par fue sumergido en agua de mar. Para cada experiencia los metales fueron pesados previamente para determinar su velocidad de corrosión, según la norma ISO 9226.

Durante el ensayo se realizaron medidas diarias de potencial de corrosión a los metales unidos y por separado, por un periodo de 16 días. Para ello se empleó un potenciostato conectado a un electrodo de referencia de calomel saturado y al electrodo de trabajo, representado tanto por el par galvánico como por el metal.

\section{RESULTADOS}

\section{Ensayos electroquímicos}

En la Figura 1 se muestra la variación del potencial de corrosión en función del tiempo para los distintos materiales en agua de mar. Una de las propiedades que debe cumplir el material utilizado como ánodo de sacrificio es tener un potencial de corrosión menor que el del metal a proteger (cátodo). Desde este punto de vista, se puede observar que tanto las aleaciones base $\mathrm{Al}$ como el cinc cumplen con esta condición (Fig. 1). También, este potencial debe tener una diferencia considerable con respecto al potencial del cátodo (por lo menos $250 \mathrm{mV}$ ), de manera de asegurarse que la aleación que actúa como ánodo se disolverá activamente en el medio. Esta diferencia la presenta el cinc y en parte la aleación de Al-Zn-In.

Además, para que el material tenga la capacidad de funcionar como ánodo debe presentar disolución activa y en un grado menor, la formación de productos de corrosión en su superficie. Curvas típicas de disolución activa la presentan la aleación de Al$\mathrm{Zn}$-Sn y el $\mathrm{Zn}$, donde se observa inicialmente una disminución de potencial de corrosión para alcanzar estabilidad en el tiempo. En cambio, la aleación AlZn-In presenta un incremento de potencial a partir de los $40 \mathrm{~min}$ lo que es propio de la formación de óxido superficial.

En la Figura 2 se presentan las curvas de polarización para los materiales estudiados obtenidas a una velocidad de barrido $\left(\mathrm{V}_{\mathrm{b}}\right)$ de $25 \mathrm{mV} \cdot \mathrm{min}^{-1}$, donde la intercepción de la curva catódica del Al NV-5083 (casco) con la curva anódica de cada una de las otras 
muestras (ánodo), entrega como resultado el potencial de corrosión y la corriente de corrosión del ánodo que forma parte del par galvánico (Tabla 2).

\section{Ensayos de protección catódica}

Los resultados de la variación del potencial en función del tiempo para los ensayos de inmersión en agua de mar de los respectivos pares galvánicos, muestran en todos los casos una diferencia de potencial entre el Al NV-5083 (casco) y el par galvá- nico, como también entre el par y el ánodo (Fig. 3). Además, en la Tabla 3 se presenta la velocidad de corrosión para las muestras galvánicas calculada a partir de la siguiente expresión:

$$
\mathrm{V}_{\mathrm{c}}=\frac{\Delta \mathrm{g}}{\mathrm{A} \cdot \mathrm{t}}
$$

donde $\Delta \mathrm{g}$ es la pérdida en peso en miligramos de la muestra, A es el área expuesta del material en $\mathrm{cm}^{2} \mathrm{y}$

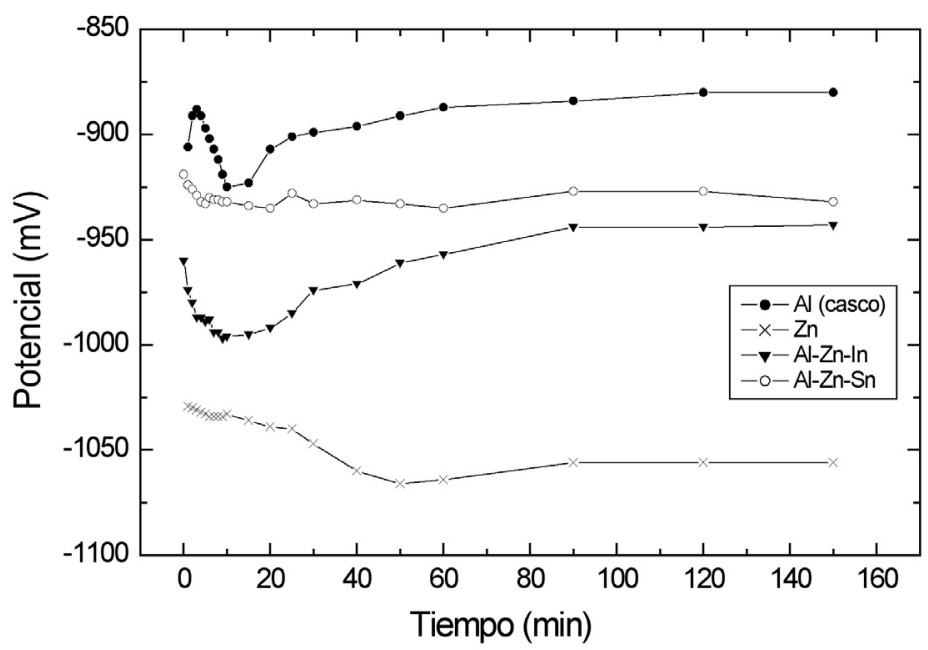

Figura 1. Potencial de corrosión en función del tiempo para Al NV-5083, aleaciones base Al y Zn en agua de mar.

Figure 1. Corrosion potential as function of time for $\mathrm{Al} \mathrm{NV-5083,} \mathrm{Al}$ alloy and $\mathrm{Zn}$ in seawater.

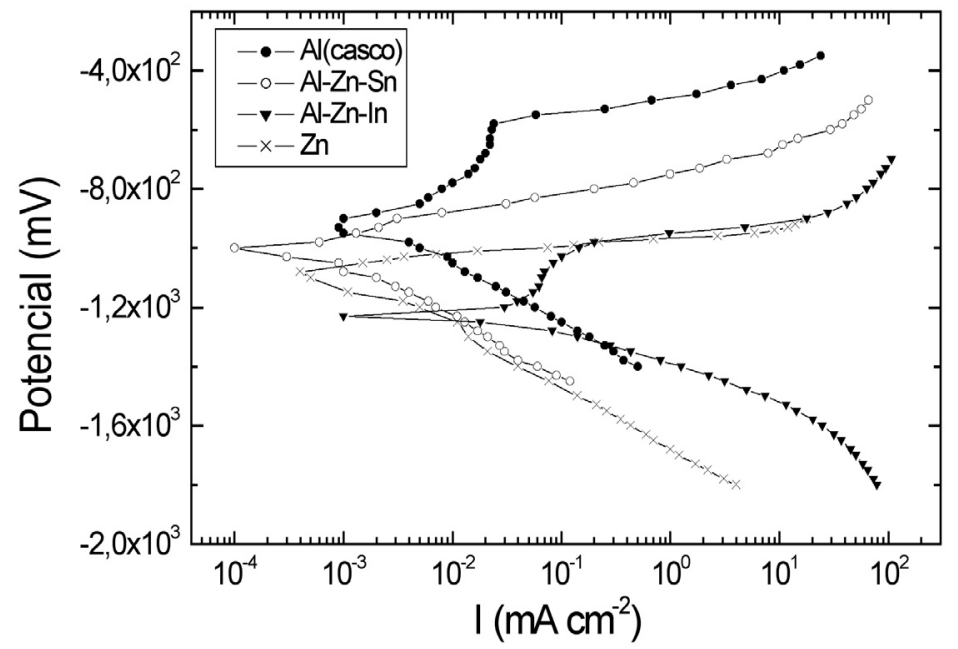

Figura 2. Curvas de polarización en condiciones aireadas para Al NV-5083, aleaciones base Al y Zn en agua de mar. $\mathrm{V}_{\mathrm{b}}: 25 \mathrm{mV} \cdot \mathrm{min}^{-1}$

Figure 2. Polarization curves in aerated condition for $\mathrm{Al} \mathrm{NV-5083,} \mathrm{Al}$ alloy and $\mathrm{Zn}$ in seawater. $\mathrm{V}_{\mathrm{b}}: 25 \mathrm{mV} \cdot \mathrm{min}^{-1}$ 
Tabla 2. Potencial (Ec), corriente (Ic) y velocidad de corrosión (Vc) del ánodo en el par galvánico.

Table 2. Potential (Ec), current (Ic) and corrosion rate (Vc) of the anode in the galvanic couple.

\begin{tabular}{cccc}
\hline Par galvánico & $\begin{array}{c}\text { Ec } \\
(\mathbf{m V})\end{array}$ & $\begin{array}{c}\text { Ic } \\
\left(\mathbf{A} \cdot \mathbf{c m}^{-2}\right)\end{array}$ & $\begin{array}{c}\text { Vc (ánodo) } \\
\left(\mathbf{m m} \cdot \mathbf{a n ̃ o} \mathbf{0}^{-1}\right)\end{array}$ \\
\hline Al NV-5083/Al-Zn-In & -1182 & $4,6 \times 10^{-5}$ & 0,500 \\
Al NV-5083/Al-Zn-Sn & -960 & $1,2 \times 10^{-6}$ & 0,013 \\
Al NV-5083/Zn & -1018 & $7,5 \times 10^{-6}$ & 0,082 \\
\hline
\end{tabular}

t es el tiempo de exposición en días. En todos los casos el aluminio NV-5083 se disuelve en menor cantidad que el ánodo.

\section{DISCUSIÓN}

En forma general, tanto en los ensayos electroquímicos como en los de inmersión, los tres ánodos de sacrificio estudiados logran proteger el aluminio NV-5083 (casco de embarcaciones) en presencia de agua de mar. Sin embargo, de acuerdo a los resultados obtenidos es posible seleccionar la composición más adecuada para el ánodo en las condiciones estudiadas.

Al considerar los valores de potencial de corrosión entre el aluminio NV-5083 y el material de los respectivos ánodos, el cinc es el que presenta una mayor diferencia $(\Delta \mathrm{Ec}=$ $180 \mathrm{mV}$ ) y además, la forma de la curva obtenida es propia de un metal activo (Fig. 1). En segunda opción le sigue la aleación Al-Zn-In con un $\Delta \mathrm{Ec}=65 \mathrm{mV}$. Sin embargo, ésta presenta un comportamiento típico de un material formador de óxido, lo que no concuerda con las especificaciones de ánodos de sacrificio. Con respecto a la aleación Al-Zn-Sn su diferencia de potencial es alrededor de $50 \mathrm{mV}$, lo que disminuye su capacidad de proteger catódicamente al aluminio NV-5083.

Los resultados anteriores concuerdan con los obtenidos en las curvas de polarización donde la curva catódica del aluminio NV-5083 intercepta a la curva anódica del Zn en la zona de disolución activa y a la aleación Al-Zn-In en el inicio de la zona pasiva (Fig. 2). No obstante, la pérdida de material de la aleación es seis veces mayor que para el $\mathrm{Zn}$.

Por otra parte, un material que actúe como ánodo de sacrificio debe presentar una diferencia mínima de potencial con respecto al potencial de corrosión del par galvánico
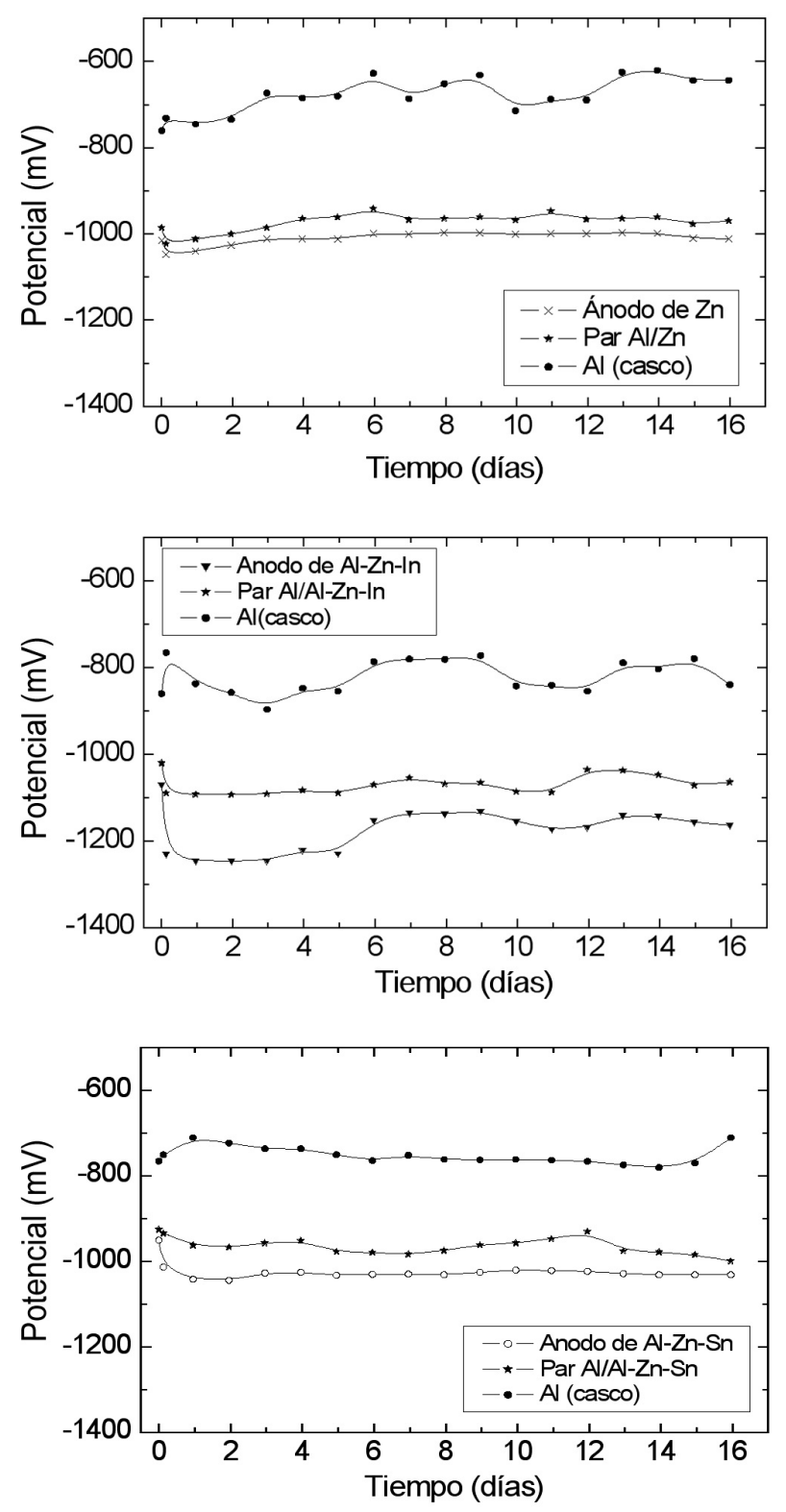

Figura 3. Potencial de corrosión en función del tiempo para los ensayos de pares galvánicos en agua de mar.

Figure 3. Corrosion potential as function of time for essays of galvanic couples in seawater. 
(ánodo-cátodo). Los resultados de la evaluación del potencial de los metales y su correspondiente par galvánico después de 16 días de ensayo, indican que el Zn cumple con los requisitos necesarios para actuar como ánodo de sacrificio: una diferencia mayor a $250 \mathrm{mV}$ entre el casco y el ánodo y además, una diferencia mínima $(\Delta \mathrm{Ec}=36 \mathrm{mV})$ entre par galvánico y ánodo (Tabla 4).

En la Figura 4 se muestra la proporción obtenida para la pérdida de peso de los distintos materiales (Tabla 3), en relación a una pérdida de aluminio NV$5083 \mathrm{de} 1,0 \mathrm{mg} \cdot \mathrm{cm}^{-2} \cdot \mathrm{día}^{-1}$. Al comparar entre estos tres ánodos se observa claramente que el $\mathrm{Zn}$ presenta un menor desgaste de material para un área igual de aluminio NV-5083, logrando un mayor tiempo de vida útil en comparación con los ánodos de base aluminio.

\section{CONCLUSIONES}

Los resultados obtenidos en esta investigación muestran que las aleaciones de base aluminio Al-Zn-In y Al-Zn-Sn presentan condiciones para proteger catódicamente al aluminio NV-5083 del casco de la embarcación. Así lo demuestran los valores obtenidos para potencial de corrosión, corriente de corrosión y pérdida de peso. Sin embargo, el cinc supera las propiedades de las aleaciones mencionadas, al presentar una menor pérdida en peso para igual área de aluminio NV-5083 y por tanto, mayor vida útil en el sistema de protección catódica.

\section{AGRADECIMIENTOS}

Los autores agradecen a la Dirección de Investigación de la Pontificia Universidad Católica de Valparaíso por el financiamiento parcial y a la Armada de Chile por su apoyo logístico.

Tabla 3. Pérdida en peso $\left(\mathrm{mg} \cdot \mathrm{cm}^{-2} \cdot \mathrm{día}^{-1}\right)$ de los pares galvánicos en el ensayo de protección catódica en agua de mar.

Table 3. Weight loss $\left(\mathrm{mg} \cdot \mathrm{cm}^{-2} \cdot \mathrm{day}^{-1}\right)$ of the galvanic couples in the essay of cathodic protection in sea water.

\begin{tabular}{ccc}
\hline Par galvánico & \multicolumn{2}{c}{ Pérdida en peso $\left(\mathbf{m g} \cdot \mathbf{c m}^{-\mathbf{2}} \cdot \mathbf{d i ́}^{\mathbf{- 1}}\right)$} \\
& Al & Ánodo \\
\hline Al NV-5083/Al-Zn-Sn & 0,012 & 0,382 \\
Al NV-5083/Al-Zn-In & 0,015 & 0,640 \\
Al NV-5083/Zn & 0,032 & 0,371 \\
\hline
\end{tabular}

Tabla 4. Potencial de corrosión promedio para el cátodo (*), ánodo (**) y par galvánico en agua de mar.

Table 4. Mean corrosion potential for the cathode (*), anode $(* *)$ and galvanic couple in sea water.

\begin{tabular}{|c|c|c|c|}
\hline & $\mathbf{E}_{\text {ecs }} / \mathbf{m V}$ & $\begin{array}{c}\text { Diferencia } \\
\text { cátodo- } \\
\text { ánodo }\end{array}$ & $\begin{array}{l}\text { Diferencia } \\
\text { par-ánodo }\end{array}$ \\
\hline *Al NV-5083 & -820 & \multirow{3}{*}{354} & \multirow{3}{*}{105} \\
\hline$* * A 1-Z n-I n$ & -1174 & & \\
\hline Par & -1069 & & \\
\hline *Al NV-5083 & -752 & \multirow{3}{*}{272} & \multirow{3}{*}{61} \\
\hline$* * \mathrm{Al}-\mathrm{Zn}-\mathrm{Sn}$ & -1024 & & \\
\hline Par & -963 & & \\
\hline *Al NV-5083 & -679 & \multirow{3}{*}{330} & \multirow{3}{*}{36} \\
\hline$* * \mathrm{Zn}$ & -1009 & & \\
\hline Par & -973 & & \\
\hline
\end{tabular}

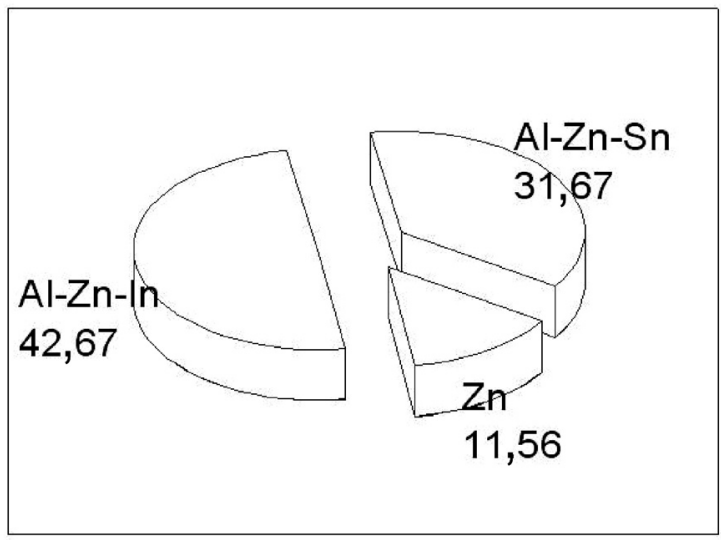

Figura 4. Pérdida de peso $\left(\mathrm{mg} \cdot \mathrm{cm}^{-2} \cdot\right.$ día $\left.^{-1}\right)$ de los ánodos y de aluminio NV-5083 en agua de mar.

Figure 4. Weight loss $\left(\mathrm{mg} \cdot \mathrm{cm}^{-2} \cdot \mathrm{dí}^{-1}\right)$ of anodes and aluminum NV-5083 in seawater. 


\section{REFERENCIAS}

Batt, M.J. 2002. Cathodic protection requirements for high strength steel in sea water assessed by potentiostatic weight loss measurements. Brit. Corros. J., 37: 31-36.

Benedetti, L., P.L. Bonora, R. Pasinetti \& S. Rossi. 1998. Laboratory and field characterization of new sacrificial anode for cathodic protection of offshores structures. Natl. Ass. Corros. Eng., 52: 1018-1023.

Bradford, S. 1993. Corrosion Control. Van Nostrand Reinhold, Toronto, vol. 1: 1-32.

Breslin, C.B., W.M. Carrol \& L.P. Friery. 1991. Influence of impurity elements on electrochemical activated by indium. J. Sci. Eng. Corros., 49(11): 895-902.

British Standards Institution. 1996. Cathodic protection. British Standards Institution, London, $100 \mathrm{pp}$.

Bessone, J.B. \& D.R. Salinas. 1991. Electrochemical behavior of $\mathrm{Al}-5 \% \mathrm{Zn}-0.1 \% \mathrm{Sn}$ sacrificial anode in aggressive media: Influence of its alloying elements and the solidification structure. J. Sci. Eng. Corros., 47(9): 665-673.

Cabrera, G.J., M.E. Casas \& B.L. Sadianes. 1993. Ánodo de sacrificio para la protección catódica. Cent. Invest. Quím. (CU), 11(21): 1-5.

Cordero, A. \& L. De Paula. 1997. Proteçao catódica, técnica de combate à corrosão. Editora Técnica, Río de Janeiro, pp. 87-98.

Eliécer, D. \& A. Antonyraj. 2001. Inusual behavior of magnesium and $\mathrm{ZM}$ anodes in aqueous electrolytes at high concentrations. Corrosion, 57(4): 334-345.
Gundersen, R. \& K. Nisancioglu. 1990. Cathodic protection of aluminum in seawater. J. Sci. Eng. Corros., 46(4): 279-285.

Latona, N., P. Fetherston, A. Chen, K. Sridharan \& R. Dodd. 2001. Wear-corrosion comparisons of passivating vs nonpassivating alloys in aerated $3.5 \%$ aqueous solutions of sodium chloride corrosion. Corrosion, 57(10): 884-888.

Maguire, R.J. \& J.A.J. Thompson. 1996. Proceedings of the workshop on organotin compounds in the Canadian aquatic environment. National Water Research Institute, 2: 96-153.

Martínez, A. 1998. Answer to CP of aluminum structures Al6061. Forum: Cathodic protection, use of zinc anodes on sub-sea aluminum structures. National Association Corrosion Engineers, Cathodic Protection 5050.

Raja, V.S. \& A. Venugopal. 1996. Effect of grainboundary corrosion on impedance characteristics of an aluminum-zinc-indium alloy in 3,5\% sodium chloride solution. J. Sci. Eng. Corros., 52(2):138142 .

Schramuk, J. \& D. Klopper. 2005. A sacrificial anode retrofit program for existing cast iron distribution water mains. Mater. Perform., 44(5): 20-24.

Suriya, U., P. Halleck, A. Grader \& J.M. Andresen. 2005. Anode butt cores: physical characterization and reactivity measurements. J. Mater., 37(2): 3540.

Tighe-Ford, D. J. \& J. S. Dahele. 2000. Ship impressed current cathodic protection modulations of system current outputs by propeller/shaft rotation on physical scale. Corrosion, 35(4): 269-272.

Uruchurtu, J. 1991. Electrochemical investigations of activation mechanism of aluminum. J. Sci. Eng. Corros., 47(6): 472-479. 